\title{
Comparison of transtibial and transportal techniques in drilling femoral tunnels during anterior cruciate ligament reconstruction using 3D-CAD models
}

\author{
This article was published in the following Dove Press journal: \\ Open Access Journal of Sports Medicine \\ 4 April 2014 \\ Number of times this article has been viewed
}

\section{Yasutaka Tashiro' \\ Ken Okazaki' \\ Munenori Uemura ${ }^{2}$ \\ Kazutaka Toyoda ${ }^{2}$ \\ Kanji Osaki \\ Hirokazu Matsubara' \\ Makoto Hashizume ${ }^{2}$ \\ Yukihide Iwamoto' \\ 'Department of Orthopaedic Surgery, ${ }^{2}$ Department of Advanced Medical Initiatives, Kyushu University \\ Hospital, Fukuoka, Japan}

Purpose: The purpose of this study was to assess the differences in bone tunnel apertures between the trans-accessory medial portal (trans-AMP) technique and the transtibial (TT) technique in double-bundle anterior cruciate ligament reconstruction. The extent of ovalization and the frequency of overlap of the two tunnel apertures were compared.

Methods: The simulation of femoral tunnel drilling with the TT and the trans-AMP techniques was performed using three-dimensional computer aided design models from two volunteers. The incidence angle of drilling against the intercondylar wall, the femoral tunnel position, the ovalization, and the overlap were analyzed. The aperture and location of the tunnels were also examined in real anterior cruciate ligament reconstruction cases $(n=36)$.

Results: The surgical simulation showed that a lower drill incident angle induced by the TT technique made the apertures of two tunnels more ovalized, located anteromedial tunnels in a shallower position to prevent posterior wall blow out, and led to a higher frequency of tunnel overlap. The trans-AMP group had tunnel places within the footprint and had less ovalization and overlap. The results of analysis for tunnels in the clinical cases were consistent with results from the surgical simulation.

Conclusion: In the TT technique, the shallow anteromedial tunnel location and more ovalized tunnel aperture can lead to a higher frequency of tunnel overlap. Compared with the TT technique, the trans-AMP technique was more useful in preparing femoral tunnels anatomically and avoiding tunnel ovalization and overlapping in double-bundle anterior cruciate ligament reconstruction.

Keywords: anterior cruciate ligament, transtibial, transaccessory medial portal, computer aided design, surgical simulation, tunnel aperture

\section{Introduction}

The anterior cruciate ligament (ACL) plays an important role in knee functions. It also stabilizes the tibial rotation and limits the anterior tibial translation. ${ }^{1,2}$ An ACL injury causes knee symptoms of instability, which can lead to meniscal tears or chondral injury, as well as osteoarthritis. ${ }^{3}$ Reconstruction of the ACL is recommended for athletes wishing to return to sports, but previous studies have suggested that the rotatory instability sometimes remained after nonanatomic reconstruction. ${ }^{4,5}$ Recently, anatomic double-bundle ACL reconstruction, which reproduces the main two bundles of ACL fibers, the anteromedial (AM) bundle and posterolateral (PL) bundle, has been advocated because several clinical studies showed that the double-bundle is more
Correspondence: Yasutaka Tashiro Department of Orthopaedic Surgery, Kyushu University, 3-I-I Maidashi, Higashi-ku, Fukuoka 8I2-8582, Japan

$\mathrm{Tel}+8 \mathrm{I} 926425488$

Fax +8I 926425507

Email Ilyasu@med.kyushu-u.ac.jp 
advantageous for restoring rotatory stability than singlebundle reconstruction. ${ }^{6-9}$

The location of the bone tunnels and the adequate graft fixation are two of the most important facets of ACL reconstruction required to achieve successful results. ${ }^{4,10,11}$ The lateral intercondylar ridge, also known as the "resident's ridge", is an important landmark during arthroscopic ACL reconstruction, as demonstrated by several cadaveric studies. ${ }^{12-15}$ However, malposition of the tunnels can occasionally occur.

Recent studies about ACL reconstruction have reported that the femoral tunnel placement on the anatomical ACL footprint performed using the traditional transtibial (TT) technique is more difficult than that by using tibial tunnelindependent techniques. ${ }^{16-19}$ Furthermore, even if surgeons are very careful to locate the two femoral bone tunnels anatomically, they sometimes encounter a relatively small footprint area and find it difficult to preserve the stable bony bridge between the two intra-articular apertures. ${ }^{20,21}$ The bone tunnel aperture can change to a more oval shape rather than a round one because of the drill insertion at an oblique angle, and as a result, the diameter in the long axis will become larger than that of the used drill. Accordingly, the overlap of those two holes can occur and lead to graft fixation failure. Failure of tunnel preparation and placement is associated with poor clinical outcomes, and it is incumbent on the operating surgeon to be diligent during these portions of the procedure.

In this study, the risk of femoral tunnel malposition and overlap during double-bundle ACL reconstruction was focused on. The purpose was: 1) to simulate the bone tunnel drilling procedure with the TT and the trans-accessory medial portal (trans-AMP) techniques using a three-dimensional (3D) computer-aided design (CAD) model of the knee and to assess the differences in tunnel apertures obtained using those two techniques; and 2) to confirm this theoretical difference on actual double-bundle ACL reconstructed knees.

Surgical simulation was used because it makes it possible to compare two different techniques in identical cases under the same conditions, and this merit is not necessarily obtained in cadaveric or in vivo studies. We hypothesized that the trans-AMP technique would be more useful to avoid the failure of tunnel preparation in double-bundle ACL reconstruction.

\section{Materials and methods}

Our research consisted of two studies. In study 1, we performed the surgical simulation of the drilling procedure.
In study 2, we conducted a postoperative bone tunnel evaluation of an actual ACL reconstruction.

\section{Study I}

A young adult male and a female with intact knees were recruited. A horizontal open magnetic resonance imaging (MRI) at 0.4 tesla (T) $\left(\right.$ Aperto $^{\circledR}$, Hitachi Medical Co, Tokyo, Japan) was applied to reproduce the knee flexion angle in ACL reconstruction. Their knees were flexed at $90^{\circ}$ for the simulation of the TT technique, and at $135^{\circ}$ for the simulation of the trans-AMP technique. ${ }^{22}$ A goniometer with the nonmagnetic material was used to ensure the flexion angles. The 3D MRI-based knee models containing bone and articular cartilage were created from the series of $1 \mathrm{~mm}$ slices twodimensional contours using the $3 \mathrm{D}$ reconstruction algorithm. The 3D knee models with the stereolithography (STL) file format were loaded onto the 3D processing software program, Mimics (Materialise, Leuven, Belgium), and a simulated drilling procedure for the bone tunnels in ACL reconstruction was performed (Figure 1A).

The tunnel position was determined according to the ligament fibers depicted on the MRIs and the anatomical footprint information based on a previous cadaveric study. ${ }^{15}$ In the simulation of the TT technique with $90^{\circ}$ knee models, the femoral AM tunnel was drilled through the tibial AM tunnel, and the PL tunnel was drilled through the tibial PL tunnel as the standard method, and then another method drilling the femoral AM tunnel through the tibial PL tunnel was also simulated.

In the simulation of the trans-AMP technique with $135^{\circ}$ knee models, the portal was established $2 \mathrm{~cm}$ medial to the patellar tendon edge, and just above the medial meniscus. In both techniques, virtual bone tunnels with a diameter of $6 \mathrm{~mm}$ (5.5 $\mathrm{mm}$ in females) for the AM and PL bundles were drilled, and the occurrence of tunnel aperture overlap and other assumable complications was surveyed. The incidence angle of virtual drilling against the femoral intercondylar wall and the diameter of virtual bone tunnel holes were measured using the 3D software package (Figure 1B). The percentage of tunnel aperture ovalization was calculated from the diameter in the long axis.

\section{Study 2}

In this study, 36 subjects with arthroscopic double-bundle ACL reconstructed knees participated in the present study. Twelve males and 24 females with a mean age of 30 years (range, 13-48) were included. The exclusion criteria included any previous reconstructive surgery on the same knee. 

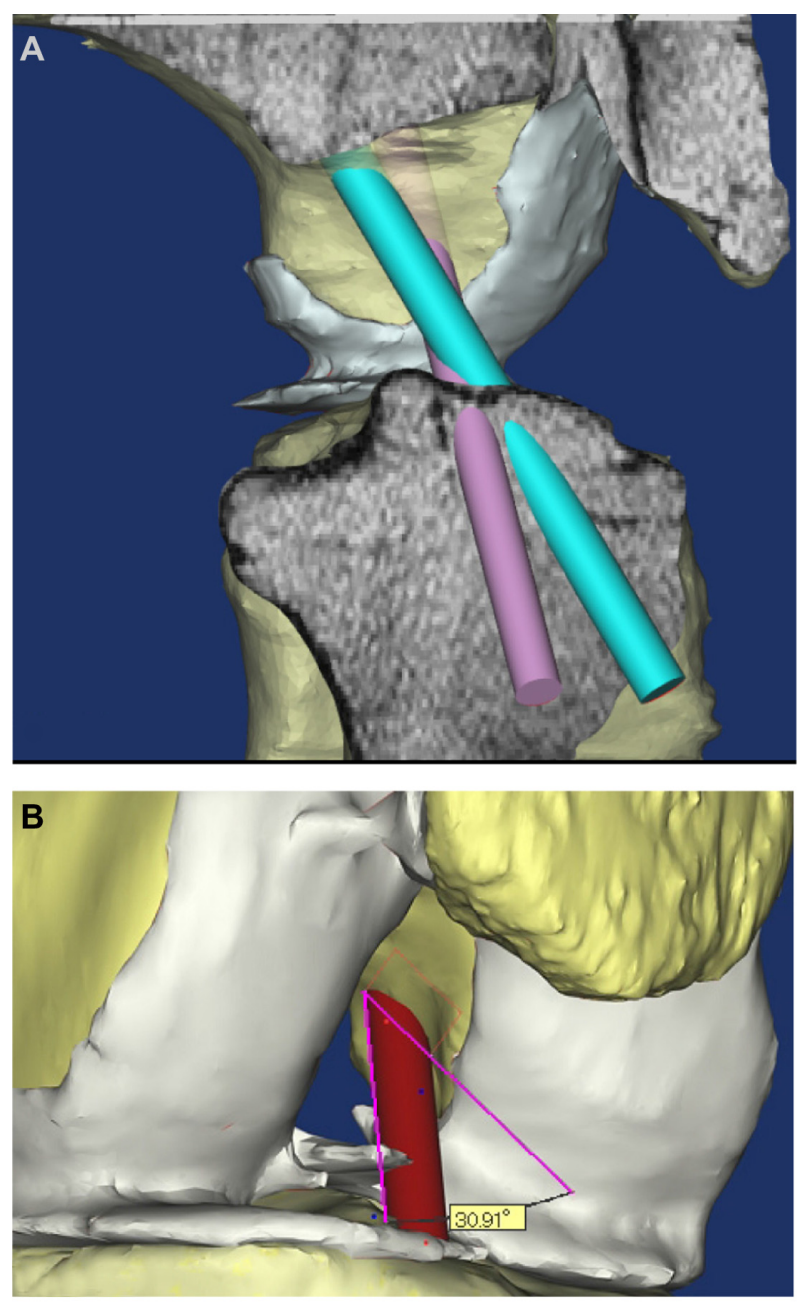

Figure I Surgical simulation.

Notes: (A) Double-bundle ACL reconstruction for a 3D MRI-based knee model is shown. Femoral tunnels were drilled using the transtibial technique in this model. (B) Incidence angle of the virtual drill against the femoral intercondylar wall was measured using the 3D software package (Mimics; Materialize, Leuven, Belgium). Abbreviations: $\mathrm{ACL}$, anterior cruciate ligament; 3D, three dimensional; MRI, magnetic resonance imaging.

The subjects underwent arthroscopic double-bundle reconstruction of the ACL of the injured knee using 5.0-7.0 mm semitendinosus tendon grafts. The gracilis tendon was additionally harvested if needed. Femoral bone tunnels for the AM bundle and PL bundle were drilled using the TT technique with the knee resting in $90^{\circ}$ flexion $(n=11)$, or the trans-AMP technique with the knee bent to $135^{\circ}$ flexion ( $\left.\mathrm{n}=25\right)$.

The resident's ridge on the femur was ascertained arthroscopically through the medial portal in all cases, and two tunnels for the AM and PL bundles were centered at the rear area of the ridge. The AM tunnels were targeted to be approximately $5 \mathrm{~mm}$ anterior to the posterior bony edge of the intercondylar wall, and the PL tunnels were targeted to be approximately 8-9 mm apart from the center of the AM aperture. The tibial tunnels for the AM and PL bundles were created in the center of the ACL footprint, avoiding impingement during knee extension. A computed tomography (CT) scan was performed 2 weeks after the operation with $0.67-1 \mathrm{~mm}$ slices. The 3D knee models were reconstructed from the CT dataset, and the lateral half-cross sections were obtained using the 3D DICOM software package (Real INTAGE, Cybernet Systems Co, Ltd, Tokyo, Japan) (Figure 2).

The bone tunnel position was measured according to the quadrant method..$^{23}$ The distance between the centers of the AM and PL tunnel holes, the occurrence of tunnel overlap, and the percentage of tunnel hole ovalization in the long axis were evaluated in both the TT group and the transAMP group. We chose a CT scan rather than the MRI for the postoperative assessment, because $3 \mathrm{D}$ reconstructions of bone tunnels and their measurements were more accurate with CT models than MRI models, which could be affected by postoperative reactive changes.

This study protocol was reviewed and approved by the institutional review board at our university, and all patients and volunteers gave their informed consent before they were included.

\section{Data analysis}

To determine the differences in the tunnel position and ovalization percentage between the two groups, we used the

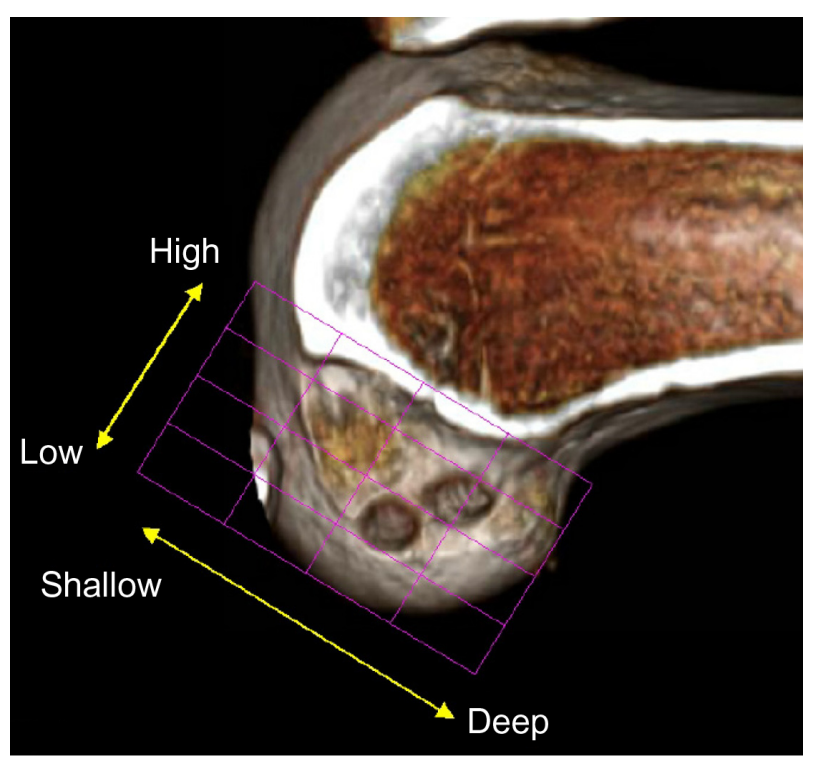

Figure 2 3D CT-based model.

Notes: Femoral bone tunnels after a double-bundle ACL reconstruction are shown. Tunnel position was assessed according to the quadrant method. ${ }^{23}$ Distance between the AM and PL tunnel centers, occurrence of tunnel overlap, and percentage of tunnel hole ovalization in the long axis were all evaluated using the 3D software program (Real INTAGE; Cybernet Systems Co, Ltd, Tokyo, Japan).

Abbreviations: 3D, three dimensional; $\mathrm{CT}$, computed tomography; $\mathrm{ACL}$, anterior cruciate ligament; AM, anteromedial; PL, posterolateral. 
unpaired Student's $t$-test in study 2. A two-tailed value of $P \leq 0.05$ was considered to be statistically significant. The analysis for the prevalence of reconstructed knees with tunnel overlap was completed using Fisher's exact test, with a value of $P \leq 0.05$ considered to indicate a significant difference.

To examine the reproducibility of this method, we randomly selected ten knees from the CT models and repeated all measurements three times by three different observers who were blinded to the results reported by the other observers, and the intraclass/interclass correlation coefficients (ICCs) were assessed. ${ }^{24} \mathrm{~A}$ repeated one-factor analysis of variance was performed to calculate the intraobserver reliability (ICC [1.3]) of the data recorded for the three measurements. The interobserver reliability (ICC [2.3]) was calculated using an unrepeated two-factor analysis of variance of the averages of the three measurements obtained by each of the three observers. The reliability of the measurement values obtained in the CT knee models ranged from good to great. The ICC (1.3) values were 0.92 and 0.94; the ICC (2.3) values were 0.87 and 0.83 for the tunnel location and tunnel diameter, respectively.

\section{Results}

\section{Study I}

In the simulation of drilling bone tunnels with the TT technique in $90^{\circ}$ flexed knees, if the anatomical femoral footprint of the AM bundle was targeted from the tibial AM tunnel, a blow out of the femoral posterior cortex occurred in both knee models. Alternatively, to avoid a blow out, the femoral AM tunnel has to be shifted to a shallower position. In addition to this change, the aperture of tunnel was ovalized, and the overlapping of tunnels occurred in both knee models (Figure 3A). When the trans-AMP technique was simulated, no blow out of the femoral posterior cortex or overlap of bone tunnels occurred in either knee model (Figure 3B). The incidence angle of virtual drills was greater in the trans-AMP technique than that in the TT technique for both the AM and PL tunnels in all cases $(P<0.01)$ (Table 1$)$. The diameters of the virtual bone tunnels were elliptically enlarged with the TT technique, while these values using the trans-AMP technique were closer to round $(P<0.01)$. When the femoral AM tunnel was drilled through the tibial PL tunnel, the drill incidence angles were slightly higher than those of the standard TT technique, but they were still smaller than the angles of the trans-AMP technique (Table 1).

\section{Study 2}

Graphic data about the tunnel position are presented in Figure $4 \mathrm{~A}$ and $\mathrm{B}$. The results from the postoperative CT

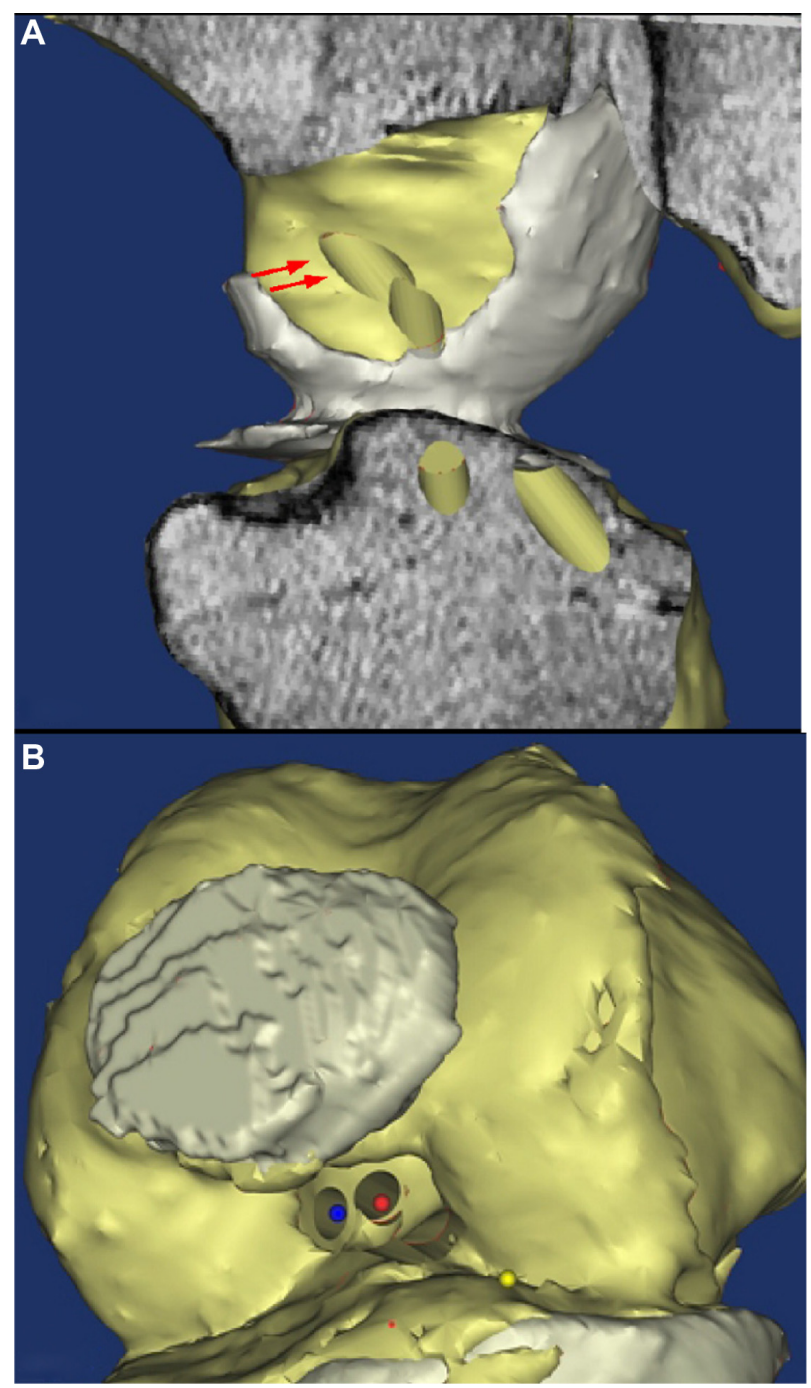

Figure 3 Simulations of femoral tunnel drilling.

Notes: (A) Femoral tunnels drilled using the transtibial technique with a $90^{\circ}$ flexed knee model is shown. AM tunnel was shifted to a shallower position (red arrows), was deformed (ovalized), and overlapped with the PL tunnel. (B) Transaccessory medial portal technique with a $135^{\circ}$ flexed knee model is shown. The red mark is the AM center, the blue one is the PL center and the yellow one is transaccessory medial portal. Tunnel ovalization was less extensive, and the bony bridge between apertures was preserved.

Abbreviations: AM, anteromedial; PL, posterolateral.

knee models are shown in Table 2. The AM tunnel was significantly shallower in the TT group $(P<0.01)$, while most of the tunnels drilled with the trans-AMP technique were located within the anatomical footprint. With respect to the PL tunnel location, no significant difference was observed between the TT group and the trans-AMP group. An overlap of tunnels occurred more often in the TT group (45\%) than in the trans-AMP group $(8 \%)(P<0.01)$, and the distance between tunnel centers in overlapped cases was significantly closer (TT, $5.9 \pm 1.2 \mathrm{~mm}$; trans-AMP, $6.0 \pm 0.5 \mathrm{~mm}$ ) than in not overlapped cases (TT, 9.6 $\pm 2.2 \mathrm{~mm}$; trans-AMP, $8.6 \pm 1.3 \mathrm{~mm}$ ). The bone tunnels in the TT group were more elliptically enlarged than those in the trans-AMP group in both the AM 
Table I Results of the simulated drilling of femoral tunnels on MRI-based knee models (Study I)

\begin{tabular}{|c|c|c|c|c|c|c|}
\hline & \multicolumn{4}{|c|}{ TT technique } & \multicolumn{2}{|c|}{ trans-AMP technique } \\
\hline & \multicolumn{2}{|c|}{ Standard } & \multicolumn{2}{|c|}{ AM drilled through tibia-PL tunnel } & \multirow[t]{2}{*}{ Angle } & \multirow[t]{2}{*}{ Ovalization } \\
\hline & Angle & Ovalization & Angle & Ovalization & & \\
\hline \multicolumn{7}{|l|}{ Case I } \\
\hline AM tunnel & $35^{\circ}$ & $183 \%$ & $37^{\circ}$ & $164 \%$ & $44^{\circ}$ & $141 \%$ \\
\hline PL tunnel & $40^{\circ}$ & $151 \%$ & - & - & $64^{\circ}$ & $112 \%$ \\
\hline \multicolumn{7}{|l|}{ Case 2} \\
\hline AM tunnel & $31^{\circ}$ & $192 \%$ & $38^{\circ}$ & $161 \%$ & $81^{\circ}$ & $103 \%$ \\
\hline PL tunnel & $33^{\circ}$ & $184 \%$ & - & - & $51^{\circ}$ & $128 \%$ \\
\hline
\end{tabular}

Notes: Angle, drill incidence angle to intercondylar wall. Ovalization, percentage of tunnel hole ovalization in the long axis.

Abbreviations: MRI, magnetic resonance imaging; TT, transtibial; trans-AMP, transaccessory medial portal; AM, anteromedial bundle; PL, posterolateral bundle.

tunnels (TT, $129 \%$ versus trans-AMP, $110 \% ; P<0.01$ ) and PL tunnels (TT, 119\% versus trans-AMP, 108\%; $P<0.05$ ).

\section{Discussion}

The anatomic double-bundle reconstruction of the ACL has been reported to successfully restore the knee stability in both cadaveric studies $^{25,26}$ and clinical research studies. ${ }^{6-9}$
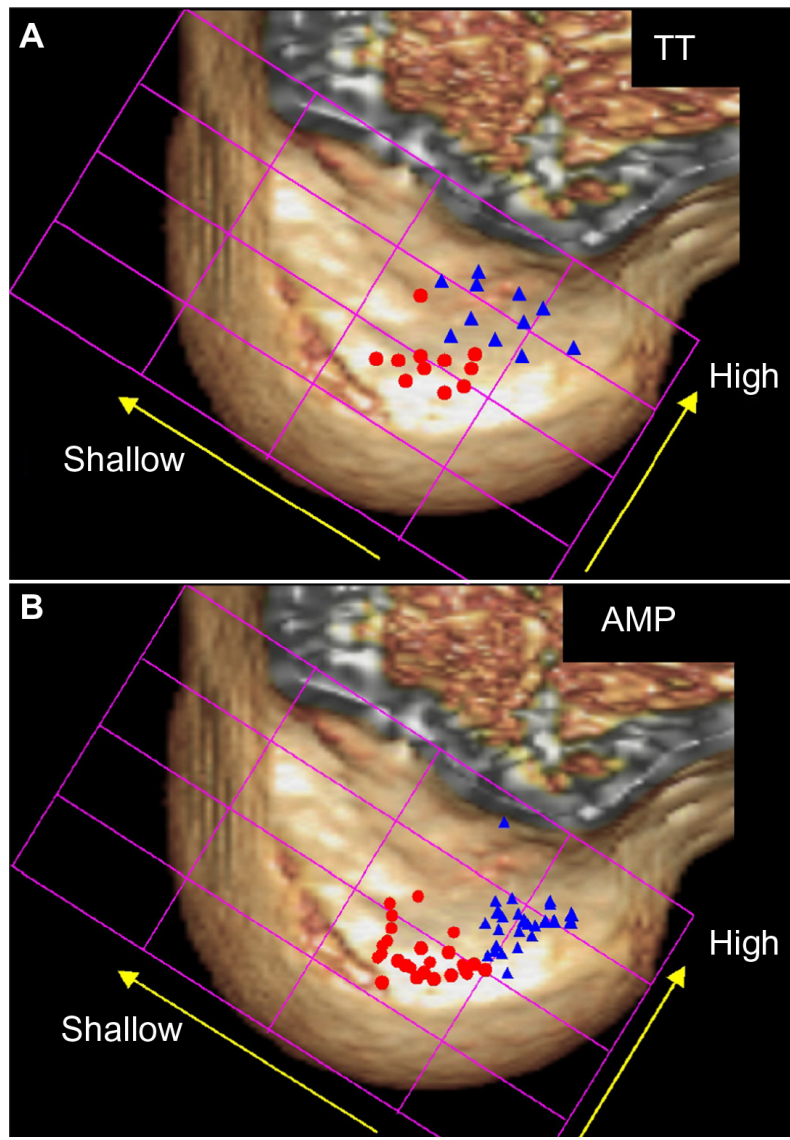

Figure 4 Postoperative tunnel positions are presented on CT knee models. Notes: Triangular dots, AM tunnels; round dots; PL tunnels. (A) TT technique positioned some AM tunnels in a shallower position than the anatomical footprint. (B) Tunnel position using the trans-AMP technique is shown. Most of the AM and PL tunnels were located within the anatomical footprint in this group.

Abbreviations: CT, computed tomography; AM, anteromedial; PL, posterolateral; $\mathrm{TT}$, transtibial; trans-AMP, transaccessory medial portal.
However, the malposition of bone tunnels or the failure of graft fixation sometimes occurs, and it can lead to a poor surgical outcome. For example, the overlap of two tunnels could worsen the strength of the graft fixation, and it should be avoided. ${ }^{27,28}$ Our study was designed to clarify the differences in femoral tunnel apertures drilled with the TT technique and the trans-AMP technique in double-bundle ACL reconstruction. We applied a medical CAD technique to simulate and assess the bone tunnel drilling procedure with the TT technique and the trans-AMP technique using a $3 \mathrm{D}$ model of the knee. The hypothesis was that there would be a difference in the risk of tunnel malposition and tunnel overlap between the two drilling procedures.

The anterior migration of the AM tunnel aperture was observed in the surgical simulation using MRI-based knee models to avoid posterior wall blow out (Study 1). The tunnel position measurement in our postoperative CT model (Study 2) also demonstrated that some femoral AM tunnels in our knees reconstructed with the TT technique were located in a shallower position than the anatomical footprint. On the other hand, most of the tunnel positions drilled through the portal were within the anatomical footprint in the

Table 2 Tunnel position, ovalization, and overlap as evaluated by postoperative CT

$\begin{array}{lll}\text { TT technique } & \begin{array}{l}\text { trans-AMP } \\ \text { technique }\end{array} & P \text {-value } \\ (\%) & \end{array}$
(\%)

\begin{tabular}{llll}
\hline AM tunnel & & & \\
Height & $26.3 \pm 7.3$ & $30.8 \pm 8.9$ & NS \\
Depth & $30.7 \pm 7.3$ & $24.4 \pm 4.2$ & $<0.0$ I \\
Ovalization & $129 \pm 25$ & $110 \pm 9$ & $<0.0$ I \\
PL tunnel & & & NS \\
Height & $49.3 \pm 8.0$ & $55.7 \pm 6.8$ & NS \\
Depth & $37.7 \pm 6.1$ & $35.8 \pm 5.6$ & $<0.05$ \\
Ovalization & $119 \pm 15$ & $108 \pm 7$ & $<0.01$ \\
Number of cases & $5(45 \%)$ & $2(8 \%)$ & \\
with tunnel overlap & & &
\end{tabular}

Abbreviations: CT, computed tomography; TT, transtibial; trans-AMP, transaccessory medial portal; AM, anteromedial bundle; PL, posterolateral bundle. 
postoperative 3D CT. A previous in vivo comparative study using high-resolution 3D MRI-based models reported that the tunnel placement using the TT technique was anterior and superior compared with that obtained using the tibial tunnel-independent technique. ${ }^{29}$

A cadaveric study that compared TT and trans-AMP reaming also reported that the tip of the guide wire - when using the TT technique - was anterior and superior to the center of the femoral footprint, but it was placed in the exact footprint when using the trans-AMP technique. ${ }^{30}$ Targeting the AM tunnel center exactly at the anatomical footprint using the TT technique would increase the risk of posterior wall blow out, so surgeons might need to divert the target slightly forward to avoid such risks when they use that technique. This consideration was supported by our MRI-based model simulation.

Drill insertion at a low oblique angle leads to an ovalization of the tunnel hole and can lead to tunnel overlap. Theoretically, the lower the incidence angle of the drill $(\theta)$ becomes, the longer the long axis of the ellipse (L) will change compared to the drill diameter (d). This relationship is reflected by the following equation:

$$
\mathrm{L}=\mathrm{d} / \sin \theta \text {. }
$$

A cadaveric study evaluating the volume and shape of the femoral intra-articular aperture with a single-bundle ACL reconstruction reported that the aperture of the femoral tunnel did indeed form an ellipse in all cases, and the percentage of ovalization in the long axis was $121 \% \pm 8 \%$ using the TT technique. ${ }^{31}$ The postoperative CT measurement of our surgically treated cases revealed a longer ellipse of the AM (129\%) and PL (119\%) tunnel holes when using the TT technique than the trans-AMP technique (AM, $110 \%$; PL, 108\%). We considered that this was due to the differences in the drill incidence angles. The results of the simulation study with relatively lower incidence angles and a longer ellipse associated with using the TT technique would support this finding, although the results from the simulation were more exaggerated than the real surgeries. The reason for the discrepancy in the results for the TT technique observed between the simulation and real surgery was that the tunnels were located at a shallower position in the real surgery. A more ovalized aperture of the femoral tunnels would lead to overlap, together with the initial closeness of the tunnels, and could worsen the graft fixation.

Previous research on anatomical ACL footprint in twenty cadaveric knees reported that the distance between the center of the AM and the PL bundle ranged from $8-10 \mathrm{~mm} \cdot{ }^{15}$ As much as $120 \%-130 \%$ elongation in the diameter of the tunnel aperture means that, when creating two tunnels with $6 \mathrm{~mm}$ diameter, the long axis would be elongated to $7.2-7.8 \mathrm{~mm}$ in the TT technique, while $110 \%$ elongation would remain $6.6 \mathrm{~mm}$ in the trans-AMP technique. In fact, our postoperative $\mathrm{CT}$ analysis revealed that when the two tunnel centers were within approximately $6-7 \mathrm{~mm}$, there was an overlap of apertures.

To preserve about $2 \mathrm{~mm}$ width of bony bridge and to avoid overlap, we consider that the two tunnel centers should be placed at least 8-9 $\mathrm{mm}$ apart from each other when creating two tunnels with $6 \mathrm{~mm}$ diameter. A clinical comparative study of single- and double-bundle ACL reconstruction also advocated that the PL tunnel center should be $9 \mathrm{~mm}$ apart from the AM center. ${ }^{27}$

The advantage of the TT technique is its safety and ease in understanding the intra-articular orientation. Therefore, this approach is familiar to many surgeons, and successful stability has been reported. ${ }^{32,33}$ However, this approach is associated with a wide variation in femoral tunnel positioning. Another solution for avoiding tunnel malposition and reducing ovalization might be drilling the femoral AM tunnel through the tibial PL tunnel, ${ }^{18}$ because this could avoid the need for drill insertion in severely low oblique angles. However, this procedure cannot be used when the femoral AM tunnel diameter is larger than that of the tibial PL tunnel, and the results of using this technique in our simulation were not as good as those obtained using the trans-AMP technique.

One of the limitations of our study was that the simulated surgical procedure was not exactly the same as the actual surgical technique, because the knee flexion angles for the simulation were fixed at $90^{\circ}$ or $135^{\circ}$ and did not have degrees of freedom. In the actual TT technique, however, surgeons are able to hang the lower leg freely and change the flexion/extension, adduction/abduction and internal/ external rotation and to finely control the drill tip in the targeted area. The results of the femoral tunnel aperture ovalization from our simulation study were therefore exaggerated when compared to those in the postoperative CT analysis, and this might have been due to the lack of freedom in the lower legs.

Nevertheless, the fact that the degree of ovalization and the risk of tunnel overlap are greater in the TT technique than in the trans-AMP technique is confirmed by the CT data for the real surgery. 


\section{Conclusion}

The results of the simulation using 3D CAD models indicated that, in the TT technique, the femoral AM tunnel location tends to be shifted to a shallower position to avoid blowing out the posterior wall, and a low oblique angle for drill insertion makes the aperture more ovalized. The shallow AM location and the ovalization can lead to a higher frequency of tunnel overlap. Compared with the TT technique, the trans-AMP technique is more advantageous with regard to positioning the femoral tunnels within the anatomical footprint and helps reduce the ovalization of the aperture in the long axis, thus reducing the risk of tunnel overlap. To avoid overlap, the two tunnel centers with $6 \mathrm{~mm}$ diameter should be placed at least 8-9 $\mathrm{mm}$ apart from each other. The results of our postoperative 3D-CT evaluation have confirmed the simulation results, and the trans-AMP technique is the preferred method to prepare femoral tunnels during anatomic double-bundle ACL reconstruction.

\section{Disclosure}

The authors report no conflicts of interest in this work.

\section{References}

1. Okazaki K, Miura H, Matsuda S, et al. Assessment of anterolateral rotatory instability in the anterior cruciate ligament-deficient knee using an open magnetic resonance imaging system. Am J Sports Med. 2007;35(7)1091-1097.

2. Sakane M, Fox RJ, Woo SL, Livesay GA, Li G, Fu FH. In situ forces in the anterior cruciate ligament and its bundles in response to anterior tibial loads. J Orthop Res. 1997;15(2):285-293.

3. Galway HR, MacIntosh DL. The lateral pivot shift: a symptom and sign of anterior cruciate ligament insufficiency. Clin Orthop Relat Res. 1980;147:45-50.

4. Loh JC, Fukuda Y, Tsuda E, Steadman RJ, Fu FH, Woo SL. Knee stability and graft function following anterior cruciate ligament reconstruction: Comparison between 11 o'clock and 10 o'clock femoral tunnel placement. 2002 Richard O'Connor Award paper. Arthroscopy. 2003;19(3):297-304.

5. Tashiro Y, Okazaki K, Miura H, et al. Quantitative assessment of rotatory instability after anterior cruciate ligament reconstruction. Am J Sports Med. 2009;37(5):909-916.

6. Izawa T, Okazaki K, Tashiro Y, et al. Comparison of rotatory stability after anterior cruciate ligament reconstruction between single-bundle and double-bundle techniques. Am J Sports Med. 2011;39(7):1470-1477.

7. Muneta T, Koga H, Mochizuki T, et al. A prospective randomized study of 4-strand semitendinosus tendon anterior cruciate ligament reconstruction comparing single-bundle and double-bundle techniques. Arthroscopy. 2007;23(6):618-628.

8. Yagi M, Kuroda R, Nagamune K, Yoshiya S, Kurosaka M. Doublebundle ACL reconstruction can improve rotational stability. Clin Orthop Relat Res. 2007;454:100-107.

9. Yasuda K, Kondo E, Ichiyama H, Tanabe Y, Tohyama H. Clinical evaluation of anatomic double-bundle anterior cruciate ligament reconstruction procedure using hamstring tendon grafts: comparisons among 3 different procedures. Arthroscopy. 2006;22(3):240-251.

10. Sohn DH, Garrett WE Jr. Transitioning to anatomic anterior cruciate ligament graft placement. J Knee Surg. 2009;22(2):155-160.
11. Strobel MJ, Castillo RJ, Weiler A. Reflex extension loss after anterior cruciate ligament reconstruction due to femoral "high noon" graft placement. Arthroscopy. 2001;17(4):408-411.

12. Bicer EK, Lustig S, Servien E, Selmi TA, Neyret P. Current knowledge in the anatomy of the human anterior cruciate ligament. Knee Surg Sports Traumatol Arthrosc. 2010;18(8):1075-1084.

13. Pombo MW, Shen W, Fu FH. Anatomic double-bundle anterior cruciate ligament reconstruction: where are we today? Arthroscopy. 2008;24(10):1168-1177.

14. Tsukada H, Ishibashi Y, Tsuda E, Fukuda A, Toh S. Anatomical analysis of the anterior cruciate ligament femoral and tibial footprints. J Orthop Sci. 2008;13(2):122-129.

15. Zantop T, Wellmann M, Fu FH, Petersen W. Tunnel positioning of anteromedial and posterolateral bundles in anatomic anterior cruciate ligament reconstruction: anatomic and radiographic findings. Am J Sports Med. 2008;36(1):65-72.

16. Gavriilidis I, Motsis EK, Pakos EE, Georgoulis AD, Mitsionis G, Xenakis TA. Transtibial versus anteromedial portal of the femoral tunnel in ACL reconstruction: a cadaveric study. Knee. 2008;15(5): 364-367.

17. Kopf S, Pombo MW, Shen W, Irrgang JJ, Fu FH. The ability of 3 different approaches to restore the anatomic anteromedial bundle femoral insertion site during anatomic anterior cruciate ligament reconstruction. Arthroscopy. 2011;27(2):200-206.

18. Tompkins M, Milewski MD, Brockmeier SF, Gaskin CM, Hart JM, Miller MD. Anatomic femoral tunnel drilling in anterior cruciate ligament reconstruction: use of an accessory medial portal versus traditional transtibial drilling. Am J Sports Med. 2012;40(6):1313-1321.

19. Tudisco C, Bisicchia S. Drilling the femoral tunnel during ACL reconstruction: transtibial versus anteromedial portal techniques. Orthopedics. 2012;35(8):e1166-e1172.

20. Iriuchishima T, Shirakura K, Yorifuji H, Aizawa S, Murakami T, $\mathrm{Fu} \mathrm{FH.} \mathrm{ACL} \mathrm{footprint} \mathrm{size} \mathrm{is} \mathrm{correlated} \mathrm{with} \mathrm{the} \mathrm{height} \mathrm{and} \mathrm{area} \mathrm{of} \mathrm{the}$ lateral wall of femoral intercondylar notch. Knee Surg Sports Traumatol Arthrosc. 2013;21(4):789-796.

21. Kopf S, Pombo MW, Szczodry M, Irrgang JJ, Fu FH. Size variability of the human anterior cruciate ligament insertion sites. Am J Sports Med. 2011;39(1):108-113.

22. Shino K, Horibe S, Hamada M, et al. Allograft anterior cruciate ligament reconstruction. Techniques in Knee Surgery. 2002;1(2): 78-85.

23. Bernard M, Hertel P, Hornung H, Cierpinski T. Femoral insertion of the ACL. Radiographic quadrant method. Am J Knee Surg. 1997;10(1):14-21; discussion 21-22.

24. Shrout PE, Fleiss JL. Intraclass correlations: uses in assessing rater reliability. Psychol Bull. 1979;86(2):420-428.

25. Wu C, Noorani S, Vercillo F, Woo SL. Tension patterns of the anteromedial and posterolateral grafts in a double-bundle anterior cruciate ligament reconstruction. J Orthop Res. 2009;27(7):879-884.

26. Zantop T, Herbort M, Raschke MJ, Fu FH, Petersen W. The role of the anteromedial and posterolateral bundles of the anterior cruciate ligament in anterior tibial translation and internal rotation. Am J Sports Med. 2007;35(2):223-227.

27. Aglietti P, Giron F, Losco M, Cuomo P, Ciardullo A, Mondanelli N. Comparison between single-and double-bundle anterior cruciate ligament reconstruction: a prospective, randomized, single-blinded clinical trial. Am J Sports Med. 2010;38(1):25-34.

28. Siebold R, Dehler C, Ellert T. Prospective randomized comparison of double-bundle versus single-bundle anterior cruciate ligament reconstruction. Arthroscopy. 2008;24(2):137-145.

29. Abebe ES, Moorman CT 3rd, Dziedzic TS, et al. Femoral tunnel placement during anterior cruciate ligament reconstruction: an in vivo imaging analysis comparing transtibial and 2-incision tibial tunnelindependent techniques. Am J Sports Med. 2009;37(10):1904-1911.

30. Bedi A, Musahl V, Steuber V, et al. Transtibial versus anteromedial portal reaming in anterior cruciate ligament reconstruction: an anatomic and biomechanical evaluation of surgical technique. Arthroscopy. 2011;27(3):380-390. 
31. Miller CD, Gerdeman AC, Hart JM, et al. A comparison of 2 drilling techniques on the femoral tunnel for anterior cruciate ligament reconstruction. Arthroscopy. 2011;27(3):372-379.

32. Kondo E, Yasuda K, Azuma H, Tanabe Y, Yagi T. Prospective clinical comparisons of anatomic double-bundle versus single-bundle anterior cruciate ligament reconstruction procedures in 328 consecutive patients. Am J Sports Med. 2008;36(9):1675-1687.
33. Tsuda E, Ishibashi Y, Fukuda A, Yamamoto Y, Tsukada H, Ono S. Tunnel position and relationship to postoperative knee laxity after double-bundle anterior cruciate ligament reconstruction with a transtibial technique. Am J Sports Med. 2010;38(4):698-706.

\section{Publish your work in this journal}

Open Access Journal of Sports Medicine is an international, peer-reviewed, open access journal publishing original research, reports, reviews and commentaries on all areas of sports medicine. The manuscript management system is completely online and includes a very quick and fair peer-review system.
Visit http://www.dovepress.com/testimonials.php to read real quotes from published authors. 